\title{
Fortbildung to go: Twitter als Ratgeber für Lehrkräfte
}

\author{
Sarah Schröder, Stefanie Vochatzer, Christian Fischer
}

University of Tübingen

Abstract: Digitale Weiterbildungsangebote für Lehrkräfte haben in der CoronaPandemie neuen Aufschwung erhalten. Auch auf sozialen Plattformen wie Twitter bilden sich immer mehr Lehrkräfte weiter - und das mitunter sogar erfolgreicher als in Präsenzveranstaltungen. Eine wichtige Rolle, die die Fortbildung auf sozialen Medien erst ermöglicht, spielt die fachliche Community.

In Zeiten des Social Distancing bieten soziale Plattformen den entscheidenden Vorteil, dass der Austausch auf Twitter, Facebook und Co. zu jeder Zeit und von jedem Ort aus kontaktlos möglich ist. Auf Twitter beispielsweise können Mikro-Blogging-Beiträge, sogenannte Tweets, mit maximal 280 Zeichen verfasst werden. Neben Texten können auch Links, Bilder und Videos geteilt sowie Umfragen erstellt werden. Im Gegensatz zu anderen sozialen Netzwerken verzichtet Twitter auf eine klassische Gruppenstruktur und setzt stattdessen Twitter auf sogenannte Hashtags (\#), die der Verschlagwortung dienen, und mit denen Nutzerinnen und Nutzer dem Verlauf einer Diskussion oder einer bestimmten Community gezielt folgen können.

\section{Vernetzung im \#twitterlehrerzimmer}

Twitter bietet eine Vielzahl an Vernetzungsmöglichkeiten, die einen flexiblen Zugang zu relevanten Wissensressourcen ermöglichen (Fischer, Fishman, \& Schoenebeck, 2019). Für Lehrkräfte im deutschen Raum findet der informelle Austausch hierbei insbesondere im \#twitterlehrerzimmer (auch \#twlz) statt. Wie stark der Wunsch nach Austausch und Vernetzung in den sozialen Netzwerken durch die Corona-Pandemie gestiegen ist, zeigt ein Blick in die Statistik. Kam der Hashtag \#twitterlehrerzimmer Anfang 2020 in ungefähr 10.000 
Tweets pro Monat vor, waren es in den ersten Monaten der Corona-Pandemie bereits bis zu 30.000 Tweets pro Monat (Fütterer et al., 2021). Die Vorteile sozialer Netzwerke - das kollaborative und vernetzte Arbeiten - werden mittlerweile von immer mehr Lehrkräften genutzt.

Neben dem \#twitterlehrerzimmer gibt es weitere Hashtags zur regionalen Vernetzung wie \#BayernEdu, \#EduSH oder \#digiHH sowie Hashtags für fachliche Lerngemeinschaften wie \#relichat oder \#pflichtfachinformatik. In diesen Communities können Lehrkräfte Erfahrungen austauschen, Fragen stellen, Diskussionen führen oder Anregungen für den (Online-)Unterricht geben. Daneben bietet Twitter Lehrkräften die Möglichkeit, mit anderen Akteuren im Bildungsbereich (wie Eltern, der Wissenschaft oder der Politik) zu interagieren (Rosenberg et al., 2020).

\section{Schneller und direkter Austausch}

Der Austausch über Twitter ist dabei wesentlich direkter als es klassische Fortbildungsformate sind (Weynand, Ferdinand, \& Fischer, 2020). Informationen werden zeitnah geteilt und können dadurch direkt angewandt werden. Darüber hinaus werden hierarchische Unterschiede innerhalb einer Community weniger betont, wodurch die Beteiligung an einer Diskussion leichter möglich ist. Außerdem ist der Zugang zu einer speziellen fachlichen Community auf Twitter wesentlich leichter als dies häufig vor Ort der Fall ist. Gibt es an einer Schule beispielsweise nur einen Informatiklehrer, sind die Möglichkeiten, fachliche Probleme zu besprechen, entsprechend begrenzt. Bei Twitter findet er mit dem Hashtag \#pflichtfachinformatik hingegen leicht eine passende Community.

\section{Communities of Practice auf Twitter?}

Die jeweiligen fachlichen Communities spielen bei der Verknüpfung von sozialen Medien und Formen der Fort- und Weiterbildung daher auch eine entscheidende Rolle: sie bilden sogenannte Communities of Practice (Lave, 1991). Das sind erfolgreiche Lehr- 
Lernform, die kollaborative Lernprozesse in den Vordergrund stellen. Um

Lerngemeinschaften als Communities of Practice zu klassifizieren, müssen drei Merkmale

(Domäne, Gemeinschaft und Praxis) erfüllt sein (Wenger-Trayner \& Wenger-Trayner, 2015).

Die Domäne beschreibt das gemeinsame Interessengebiet einzelner Personen, worüber die Identifikation als Gruppe ermöglicht und ein Zugehörigkeitsgefühl geschaffen wird. Auf Twitter übertragen bedeutet dies, dass sich durch Hashtags wie \#pflichtfachinformatik oder \#relichat eine fachliche Lerngemeinschaften entwickelt, die sich über ein gemeinsames Interessengebiet, wie Informatik oder Religionsunterricht mit dieser Community identifizieren. Das Kompetenzniveau der einzelnen Mitglieder ist dabei zunächst weniger wichtig. So kann sich ein Referendar mit einem bestimmten Fach ebenso identifizieren wie eine promovierte Lehrerin mit 35 Jahren Berufserfahrung. Eine Abgrenzung findet lediglich $\mathrm{zu}$ anderen fachlichen Communities statt.

Die Gemeinschaft bezeichnet die Beteiligung und Aktivität der Mitglieder innerhalb einer Lerngemeinschaft. Darunter fällt zum Beispiel die Interaktion, die Diskussionen über die unterrichtliche Praxis anregt, aber auch der Austausch von Lernmaterialien oder die Beantwortung gezielter Fragen durch andere Lehrkräfte. Auf diese Weise werden Lernprozesse angeregt, wodurch sich die Mitglieder einer Lerngemeinschaft gegenseitig fortund weiterbilden. Wichtig ist, dass einzelne Teilnehmerinnen und Teilnehmer nicht in allen Diskussionen beteiligt sein müssen, um Teil der Lerngemeinschaft zu sein. Dennoch können die flachen Hierarchien und die kollaborative Umgebung dazu beitragen, dass der Austausch auf Twitter deutlich stärker ausfällt als in der Schule. Insbesondere Lernmaterialen werden von den Mitgliedern einer Lerngemeinschaft häufig als lernförderlich wahrgenommen (Wesely, 2013). Zunächst können die vorgeschlagenen Materialien der Community dabei für den eigenen Unterricht eingesetzt werden. Anschließend werden die persönlichen Erfahrungen wiederum in die Twitter-Lerngemeinschaft eingebracht und die Diskussion an dieser Stelle fortgesetzt. 
Die Praxis bezieht sich auf die Profession einer Lehrperson. Ein Beispiel für einen professionsbezogenen Austausch auf Twitter ist das gemeinsame Erarbeiten von sogenannten Wikis oder Padlets (digitale Pinnwände) zu relevanten Themenfeldern. Dabei wird nicht nur auf das Wissen einzelner Mitglieder einer Lerngemeinschaft zurückgegriffen, sondern das Ergebnis auch allen Teilnehmenden zugänglich gemacht. Auf diese Weise können innerhalb einer Lerngemeinschaft langanhaltenden Beziehungen und Interaktionen zwischen verschiedenen Lehrpersonen entstehen - über Jahre hinweg und obwohl sich die Lehrkräfte geographisch an ganz unterschiedlichen Orten befinden.

\section{Vorteile nicht ganz ohne Risiko}

Die Beteiligung in Communities auf Twitter kann das Wissen und die Fähigkeiten von Lehrkräften somit nachhaltig erweitern. Allerdings gilt wie für alle sozialen Plattformen auch für Twitter, dass es keine gesicherten ,Qualitätsmarker` gibt. Die fachliche Richtigkeit einzelner Aussagen wird auf Systemebene nicht überprüft und Falschinformationen kursieren auf Twitter ebenso wie auf Facebook oder Instagram. Lehrkräfte sollten daher immer hinterfragen, ob Inhalte, die sie für den eigenen Unterricht in Erwägung ziehen, wirklich dem aktuellen Forschungsstand entsprechen. In der Regel erhalten qualitativ hochwertige Inhalte zwar mehr Likes und werden häufiger retweetet - eine Garantie für die Qualität des Inhalts ist das aber nicht.

\section{References}

Fischer, C., Fishman, B., \& Schoenebeck, S. Y. (2019). New contexts for professional learning: Analyzing high school science teachers' engagement on Twitter. AERA Open, 5(4), 1-20. https://doi.org/10.1177/2332858419894252

Fütterer, T., Hoch, E., Stürmer, K., Lachner, A., Fischer, C., \& Scheiter, K. (2021). Was bewegt Lehrpersonen während der Schulschließungen? - Eine Analyse der Kommunikation im Twitter-Lehrerzimmer über Chancen und Herausforderungen 
digitalen Unterrichts. Zeitschrift für Erziehungswissenschaft, 24, 443-477.

https://doi.org/10.1007/s11618-021-01013-8

Lave, J. (1991). Situating Learning in Communities of Practice. In L. Resnick, B. Levine, M.

John \& S. Teasley (Eds.), Perspectives on Socially Shared Cognition. pp. 63-82

Rosenberg, J. M., Reid, J. W., Dyer, E., Koehler, M., Fischer, C., \& McKenna, T. J. (2020).

Idle Chatter or Compelling Conversation? The Potential of the Social Media-based \#NGSSchat Network for Supporting Science Education Reform Efforts. Journal of Research in Science Teaching, 57(9), 1322-1355. https://doi.org/10.1002/tea.21660

Wenger-Trayner, E. \& Wenger-Trayner, B. (2015). Communities of practice: A brief introduction. https://wenger-trayner.com/wp-content/uploads/2015/04/07-Briefintroduction-to-communities-of-practice.pdf, retrieved March 1, 2021.

Wesely, P. M. (2013). Investigating the Community of Practice of World Language Educators on Twitter. Journal of Teacher Education, 64(4), 305-318.

Weynand, M., Ferdinand, J., \& Fischer, C. (2020). Lehrkräftefortbildung durch soziale Medien!? OSF Preprints. https://doi.org/10.31219/osf.io/qc6wx

Sarah Schröder, B.A. studiert Erziehungswissenschaft mit dem Schwerpunkt auf Sozialpädagogik und Soziale Arbeit in Tübingen.

Stefanie Vochatzer, B.A. ist Studentin im Masterstudiengang Erziehung und Bildung: KulturPolitik-Gesellschaft an der Eberhard Karls Universität Tübingen und Lehrerin in der praxisintegrierten Ausbildung für Erzieher*Innen und Kinderpfleger*Innen an der HeleneLange-Schule \& Fröbelseminar Mannheim.

Christian Fischer, Ph.D. ist Tenure-Track-Professor für Educational Effectiveness am Hector-Institut für Empirische Bildungsforschung der Eberhard Karls Universität Tübingen. 